\title{
GLI2 expression levels in radical nephrectomy specimens as a predictor of disease progression in patients with metastatic clear cell renal cell carcinoma following treatment with sunitinib
}

\author{
JUNYA FURUKAWA, HIDEAKI MIYAKE and MASATO FUJISAWA \\ Division of Urology, Kobe University Graduate School of Medicine, Kobe 650-0017, Japan
}

Received March 1, 2016; Accepted May 20, 2016

DOI: $10.3892 / \operatorname{mco} .2016 .950$

\begin{abstract}
The aim of the present study was to investigate the role of the Hedgehog signaling pathway in the progression of metastatic clear cell renal cell carcinoma (m-ccRCC) as well as the molecular targets of sunitinib, an inhibitor of multiple tyrosine kinases. A total of 39 patients subjected to radical nephrectomy who were diagnosed with m-ccRCC and were subsequently treated with sunitinib were enrolled in the present study. The expression levels of the Hedgehog signaling proteins (GLI1, GLI2, cyclin D1, cyclin E and transforming growth factor- $\beta$ ) and major molecular targets of sunitinib [vascular endothelial growth factor receptor (VEGFR)-1 and -2 , and platelet-derived growth factor receptor- $\alpha$ and $-\beta$ ] in primary RCC specimens were assessed by immunohistochemical staining. The expression levels of GLI2, VEGFR-1, VEGFR-2 and pre-treatment C-reactive protein as well as the Memorial Sloan-Kettering Cancer Center risk were identified as significant predictors of progression-free survival (PFS). Of these, only GLI2 expression was independently correlated to PFS according to multivariate analysis. Furthermore, treatment with sunitinib resulted in a marked inhibition of GLI2 expression in the parental human RCC ACHN cell line, but not in ACHN cells with acquired resistance to sunitinib. These findings suggested that GLI2 may be involved in the acquisition of resistance to sunitinib in RCC; thus, it may be useful to consider the expression levels of GLI2 in addition to conventional prognostic parameters when selecting m-ccRCC patients likely to benefit from treatment with sunitinib.
\end{abstract}

\section{Introduction}

Renal cell carcinoma (RCC) is highly resistant to conventional chemotherapeutic agents (1). Previously, cytokine

Correspondence to: Dr Hideaki Miyake, Division of Urology, Kobe University Graduate School of Medicine, 7-5-1 Kusunoki-cho, Kobe 650-0017, Japan

E-mail: hideakimiyake@hotmail.com

Key words: sunitinib, GLI2, metastatic clear cell renal cell carcinoma, progression-free survival therapies were the only available treatment approach for patients with metastatic RCC (m-RCC); however, the efficacy of these treatments was low with a median overall survival of $\sim 1$ year (2). Based on the increased knowledge of the molecular mechanisms involved in the progression of RCC, several types of novel molecular-targeted agents have been developed, and their introduction into clinical practice has resulted in a marked paradigm shift regarding therapeutic strategies for m-RCC (3).

Of several molecular-targeted agents, sunitinib, an orally available inhibitor of multiple tyrosine kinases, exhibits one of the strongest antitumor activities against m-RCC (4). In preclinical experimental studies, sunitinib has been demonstrated to exert inhibitory effects on tumor cell proliferation and angiogenesis (5). Furthermore, the significantly superior efficacy of sunitinib over interferon- $\alpha($ IFN- $\alpha)$ as a first-line therapy for $\mathrm{m}-\mathrm{RCC}$ has been demonstrated in a clinical setting, with a median progression-free survival (PFS) of 11 months, compared with 5 months compared for the IFN- $\alpha$ arm (6). However, several limitations are associated with the use of sunitinib for patients with m-RCC, including the comparatively short interval of a durable response and the low proportion of patients showing a complete response (CR) (7). In order to provide individualized risk-directed therapies for m-RCC patients, it is advantageous to identify novel markers predicting their susceptibility to sunitinib treatment.

To date, various model systems have been developed to predict the clinical course of $\mathrm{m}$-RCC patients receiving molecular-targeted agents $(8,9)$. However, it may be difficult to predict the prognosis of patients with m-RCC based on conventional clinicopathological parameters alone, since $\mathrm{RCC}$ has been characterized by unique biological features as well as heterogeneous genetic backgrounds (10). Therefore, the present study evaluated the expression levels of multiple potential molecular markers involved in the Hedgehog signaling pathway, which has been demonstrated to have an important role in the progression of a large variety of malignant tumor types via the regulation of numerous target genes (11), in addition to major molecular targets of sunitinib, in radical nephrectomy specimens from a total of 39 consecutive metastatic clear cell RCC (m-ccRCC) patients treated with sunitinib by immunohistochemical staining, and analyzed their association with the outcome based on several conventional parameters. 


\section{Patients and methods}

Patients. The present study included a total of 39 consecutive patients who underwent radical nephrectomy for ccRCC and were diagnosed with metastatic disease. These patients were subsequently treated with sunitinib as a first-line systemic therapy between April 2009 and March 2011 at Kobe University Hospital (Kobe, Japan). Informed consent was obtained from each patient prior to enrolment in the present study, and the study design was approved by the Research Ethics Committee of Kobe University Hospital.

Treatment with sunitinib. All the patients included in the present study initially received $50 \mathrm{mg}$ sunitinib once daily in repeated 6-week cycles, consisting of 4 weeks of treatment followed by a break of 2 weeks. Sunitinib was continuously administered until disease progression or intolerable adverse events (AEs). In cases with treatment-associated AEs corresponding to grade $\geq 3$, the dose of sunitinib was modified by initial dose reduction from 50 to $37.5 \mathrm{mg}$ /day and subsequently to $25 \mathrm{mg}$ /day.

Patient evaluation. As baseline evaluations, the performance status (PS) was assessed and clinicopathological examinations were performed based on the Karnofsky PS scale (12) and the Union for International Cancer Control Tumor-Nodes-Metastasis classification system (13), respectively, while risk classification was performed using the Memorial Sloan-Kettering Cancer Center (MSKCC) (8) and Heng's risk classification systems (9). Prior to the initiation of sunitinib treatment, radiological evaluations were performed for all patients by computed tomography (CT) of the brain, chest and abdomen as well as a radionuclide bone scan. In general, tumor measurements were repeated by $\mathrm{CT}$ at least every 12 weeks after the initiation of sunitinib treatment.

Immunohistochemical staining. Immunohistochemical staining of radical nephrectomy specimens was performed as previously described (14). In brief, formaldehyde-fixed, paraffin-embedded tissue sections were deparaffinized, rehydrated and incubated with 5\% normal blocking serum for $20 \mathrm{~min}$ at room temperature. The sections were incubated at $4^{\circ} \mathrm{C}$ overnight with 1:100 diluted antibodies targeting human GLI1 or GLI2 rabbit polyclonal antibody (cat. nos. ab92611 and ab26056, respectively; both from Abcam, Cambridge, MA, USA), cyclin D1 rabbit polyclonal antibody (cat. no. 2922; Cell Signaling Technology, Inc., Danvers, MA, USA), cyclin E rabbit polyclonal antibody (cat. no. sc-481; Santa Cruz Biotechnology, Inc., Dallas, TX, USA), transforming growth factor (TGF)- $\beta$ rabbit polyclonal antibody (cat. no. ab66043; Abcam), vascular endothelial growth factor receptor-1 (VEGFR-1) rabbit polyclonal antibody (cat. no. ab2350; Epitomics, Burlingame, CA, USA), VEGFR-2 rabbit monoclonal antibody (cat. no. 9698; Cell Signaling Technology, Inc.), platelet-derived growth factor receptor- $\alpha$ (PDGFR- $\alpha$ ) rabbit polyclonal antibody or PDGFR- $\beta$ rabbit polyclonal antibody (cat. nos. sc-338 and sc-432, respectively; both from Santa Cruz Biotechnology, Inc.), followed by incubation with biotinylated immunoglobulin G (Vector Laboratories, Burlingame, CA, USA). After incubation in an avidin-biotin peroxidase complex for $30 \mathrm{~min}$, the samples were exposed to diaminobenzidine tetrahydrochloride solution (Nacalai Tesque, Inc., Kyoto, Japan) and counterstained with methyl green (Wako Pure Chemical Industries, Ltd., Osaka, Japan).

Staining results were interpreted by two independent investigators blinded to the clinicopathological findings of the included patients. Discrepancies between results were resolved by joint review and/or consultation with a third investigator familiar with the immunohistochemical pathology. For each protein, the highest immunohistochemical staining intensity was visually scored in several fields of each section and classified as negative, weak, moderate or strong. According to previous studies, either moderate or strong staining intensity in $>10 \%$ of tumor cells was classified as strong expression $(15,16)$.

Western blot analysis. In our previous study, a human RCC cell line resistant to sunitinib (ACHN/R) was generated by culturing parental ACHN cells $(\mathrm{ACHN} / \mathrm{P})$ in the presence of sunitinib at serially increased doses (17). ACHN/P and $\mathrm{ACHN} / \mathrm{R}$ cells cultured for $24 \mathrm{~h}$ in either standard medium or medium containing $5 \mu \mathrm{M}$ sunitinib were lysed and equal amounts of protein $(25 \mu \mathrm{g})$ measured by the Bradford protein assay from lysates were subjected to $10 \%$ of sodium dodecyl sulfate polyacrylamide gel electrophoresis and transferred onto a nitrocellulose membrane. The membrane was then incubated at $4^{\circ} \mathrm{C}$ overnight with 1:1,000 diluted antibodies against GLI2 (Abcam) and 1:5,000 diluted $\beta$-actin (Santa Cruz Biotechnology, Inc.), and further incubated for $30 \mathrm{~min}$ with horseradish peroxide-conjugated secondary antibodies (Santa Cruz Biotechnology, Inc.). Specific proteins were detected using an enhanced chemiluminescence Western blot analysis system (GE Healthcare, Little Chalfont, UK).

Statistical analysis. All statistical analyses were performed using Statview 5.0 software (Abacus Concepts, Inc., Berkeley, CA, USA). $\mathrm{P}<0.05$ was considered to indicate a statistically significant difference. PFS rates were calculated using the Kaplan-Meier method and differences were determined by the log-rank test. The Cox proportional hazards regression model was used to assess the prognostic significance of certain factors.

\section{Results}

Patient response to sunitinib. The clinicopathological characteristics and outcomes of the $39 \mathrm{~m}$-ccRCC patients included in the present study and the expression of various molecular markers in their resected primary tumors are listed in Table I. Treatment with sunitinib achieved a CR in 1 patient, while 5 patients showed a partial response, 23 had stable disease and 10 had progressive disease. The overall response rate to sunitinib was $16.7 \%$ and the median duration of the objective response in the 6 responders was 9.4 months.

GLI2 is an independent predictor of PFS. During the follow-up period of 15.1 months from the initiation of sunitinib treatment, 26 patients $(66.7 \%)$ showed disease progression and the median duration of PFS was 13.2 months. As shown in Fig. 1, the 1- and 2-year PFS rates were 55.5 and $31.0 \%$, respectively. To identify 
Table I. Patient characteristics.

\begin{tabular}{ll}
\hline Variable & Patients \\
\hline
\end{tabular}

Age, years (range)

$61(36-77)$

Gender, n (\%)

Male

$27(69.2)$

Female

$12(30.8)$

Pathological tumor stage, n (\%)

$$
\begin{aligned}
& \text { pT1 } \\
& \text { pT2 } \\
& \text { pT3 } \\
& \text { pT4 }
\end{aligned}
$$

Tumor grade, n (\%)

$$
2
$$$$
3
$$

Microvascular invasion, n (\%)

\section{Negative}

Positive

Metastatic sites, n (\%)

Single

Multiple

MSKCC classification, n (\%)

Favorable

Intermediate

Poor

Heng's risk classification, n (\%)

Favorable
Intermediate
Poor

C-reactive protein $(\mathrm{mg} / \mathrm{dl})$ (range)

GLI1 expression, n (\%)

Weak

Strong

GLI2 expression, n (\%)

Weak

Strong

Cyclin D1 expression, n (\%)

Weak

Strong

Cyclin E expression, n (\%)

Weak

Strong

TGF- $\beta$ expression, n (\%)

Weak

Strong

$1.9(<0.1-18.3)$

25 (64.1)

14 (35.9)

$21(53.8)$

18 (46.2)

VEGFR-1 expression, n (\%)

Weak

Strong

Weak

Strong
Table I. Continued.

\begin{tabular}{lc}
\hline Variable & Patients \\
\hline Strong & $33(84.6)$ \\
PDGFR- $\beta$ expression, $n(\%)$ & \\
Weak & $13(33.3)$ \\
Strong & $26(66.7)$ \\
\hline
\end{tabular}

MSKCC, Memorial Sloan-Kettering Cancer Center; TGF, transforming growth factor; VEGFR, vascular endothelial growth factor receptor; PDGFR, platelet-derived growth factor receptor.

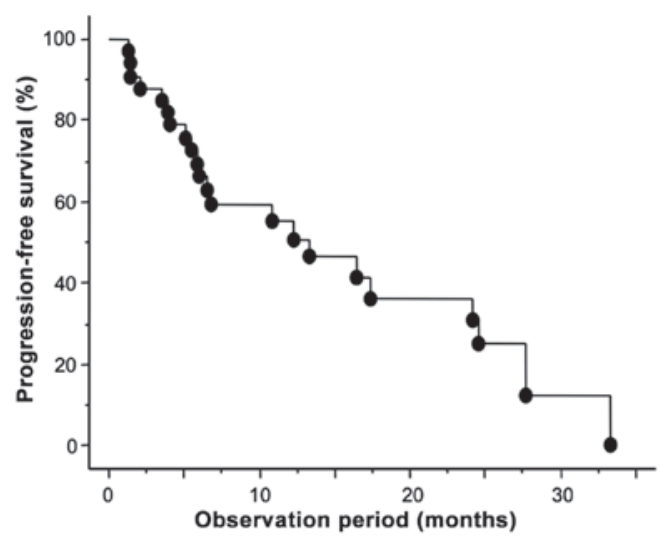

Figure 1. Progression-free survival of the 39 patients with metastatic clear cell renal cell carcinoma treated with sunitinib.

parameters associated with PFS in m-ccRCC patients treated with sunitinib, uni- and multivariate analyses were performed using the Cox proportional hazard regression model. Of the 9 molecular markers analyzed in the present study, the expression levels of GLI2, VEGFR1 and VEGFR2 were identified as significant predictors of PFS by univariate analysis (Table II). In Fig. 2, the PFS curves according to the expression status of GLI2, VEGFR1 and VEGFR2 are presented in addition to the representative immunohistochemical images for the expression levels of these molecular markers. In addition to these molecular markers, the MSKCC and baseline C-reactive protein (CRP) levels were also significantly correlated with PFS among several conventional factors examined. Furthermore, multivariate analysis of these 5 significant predictors of PFS on univariate analysis revealed that only the expression status of GLI2 was independently correlated with the other factors included (Table II).

GLI2 expression is involved in the resistance of RCC to sunitinib in vitro. To further characterize the significance of GLI2 expression in RCC tissues with regard to the efficacy of sunitinib, changes in GLI2 expression in ACHN/P and $\mathrm{ACHN} / \mathrm{R}$ cells cultured in the absence or presence of sunitinib were examined. As shown in Fig. 3, despite the lack of a significant difference in GLI2 expression between ACHN/P and $\mathrm{ACHN} / \mathrm{R}$ in the absence of sunitinib, administration of sunitinib resulted in a marked downregulation of GLI2 in ACHN/P, but not in ACHN/R. 
Table II. Uni- and multivariate analyses of the association between various parameters with progression-free survival.

\begin{tabular}{|c|c|c|c|c|}
\hline \multirow[b]{2}{*}{ Parameter } & \multicolumn{2}{|c|}{ Univariate analysis } & \multicolumn{2}{|c|}{ Multivariate analysis } \\
\hline & $\begin{array}{c}\text { Hazard ratio } \\
(95 \% \mathrm{CI})\end{array}$ & P-value & $\begin{array}{c}\text { Hazard ratio } \\
(95 \% \mathrm{CI})\end{array}$ & P-value \\
\hline Age, years (<70 vs. $\leq 70)$ & $1.50(0.65-3.4)$ & 0.33 & & \\
\hline Gender (male verses female) & $1.46(0.48-4.38)$ & 0.49 & & \\
\hline Karnofsky performance scale ( $\geq 80$ vs. $<80$ ) & $1.17(0.39-7.40)$ & 0.77 & & \\
\hline Pathological tumor stage (pT1/pT2 vs. pT3/pT4) & $2.55(0.74-8.77)$ & 0.13 & & \\
\hline Tumor grade (2 vs. 3 ) & $1.72(0.67-4.40)$ & 0.25 & & \\
\hline Microvascular invasion (negative verses positive) & $1.71(0.39-7.40)$ & 0.47 & & \\
\hline Metastatic sites (single verses multiple) & $1.14(0.47-2.73)$ & 0.76 & & \\
\hline MSKCC classification (favorable/intermediate verses poor) & $2.57(1.03-7.19)$ & 0.042 & $2.15(0.23-19.8)$ & 0.49 \\
\hline Heng's risk classification (favorable/intermediate verses poor) & $1.27(0.33-1.86)$ & 0.58 & & \\
\hline Baseline C-reactive protein (normal verses abnormal) & $2.69(1.07-6.73)$ & 0.034 & $2.24(0.68-7.43)$ & 0.18 \\
\hline GLI1 (low verses high expression) & $1.04(0.43-2.53)$ & 0.91 & & \\
\hline GLI2 (low verses high expression) & $3.57(1.33-9.52)$ & 0.011 & $3.86(1.11-13.3)$ & 0.038 \\
\hline Cyclin D1 (low verses high expression) & $2.04(0.85-4.90)$ & 0.10 & & \\
\hline Cyclin E (low verses high expression) & $1.12(0.47-2.83)$ & 0.79 & & \\
\hline TGF- $\beta$ (low verses high expression) & $1.56(0.64-3.78)$ & 0.32 & & \\
\hline VEGFR-1 (low verses high expression) & $2.69(1.10-6.58)$ & 0.029 & $5.17(0.38-69.8)$ & 0.21 \\
\hline VEGFR-2 (low verses high expression) & $2.66(1.04-6.79)$ & 0.040 & $3.55(0.23-52.6)$ & 0.35 \\
\hline PDGFR- $\alpha$ (low verses high expression) & $1.27(0.41-3.92)$ & 0.67 & & \\
\hline PDGFR- $\beta$ (low verses high expression) & $1.45(0.58-3.64)$ & 0.41 & & \\
\hline
\end{tabular}

CI, confidence interval; MSKCC, Memorial Sloan-Kettering Cancer Center; TGF, transforming growth factor; VEGFR, vascular endothelial growth factor receptor; PDGFR, platelet-derived growth factor receptor.

\section{Discussion}

Due to the results of a pivotal randomized phase-III clinical trial (6), sunitinib is currently used as standard first-line treatment of m-ccRCC. Furthermore, Gore et al (18) previously reported the acceptable efficacy and safety profiles of sunitinib in a global expanded-access trial of patients with m-RCC. Our recent retrospective study comprehensively evaluated the clinical outcomes in a total of 110 Japanese patients who received sunitinib as a first-line therapy for $\mathrm{m}$-RCC and reported encouraging findings with respect to cancer control as well as tolerability in a clinical setting (19). However, the use of sunitinib has several limitations. Therefore, the patients with $\mathrm{m}-\mathrm{RCC}$ who are likely to respond to sunitinib treatment should be selected prior to its administration.

To date, various studies have indicated the efficiency of several types of biomarker to assess the prognosis of patients with m-RCC treated with sunitinib (20). Our previous study reported that an imbalance between the serum levels of matrix metalloproteinase- 9 and tissue expression levels of inhibitors of matrix metalloproteinase-2 levels may serve as a novel biomarker to predict the disease progression in patients with $\mathrm{m}-\mathrm{RCC}$ undergoing treatment with sunitinib (21). However, to date, no such markers have been introduced into clinical practice. A number of studies have suggested the important role of the molecules associated with the Hedgehog signaling pathway in the progression of a wide variety of malignant tumor types, including RCC (11,22-24). For example, Dormoy et al (22) reported that inactivation of the Hedgehog pathway by a specific inhibitor, cyclopamine, induced the regression of ccRCC tumors in nude mice through the inhibition of tumor cell proliferation and neo-vascularization. Furthermore, D'Amato et al (23) showed the involvement of Hedgehog signaling in the resistance of RCC cells to molecular-targeted agents, including sunitinib. Considering these findings, the present study evaluated the expression levels of Hedgehog signaling-related proteins in addition to major molecular targets of sunitinib in primary tumor specimens in order to identify prognostic factors that are significantly correlated with the outcome for patients with m-ccRCC treated by sunitinib.

In the present study, a total of 39 patients with m-ccRCC who underwent radical nephrectomy and subsequently received sunitinib as a first-line systemic therapy were included. All 9 molecular markers examined were detectable by immunohistochemical staining in the majority of primary ccRCC tissues. Of these, only GLI2, VEGFR1 and VEGFR2 were identified as significant predictors of PFS on univariate analysis. Several previous studies reported the significance of VEGFR and its associated proteins as biomarkers in RCC patients treated with sunitinib $(25,26)$. For instance, Deprimo et al (25) reported that changes in plasma VEGF and VEGFR levels in patients showing an objective response to sunitinib were greater compared with those in patients with stable disease or disease progression (25). To the best of our knowledge, the 
A

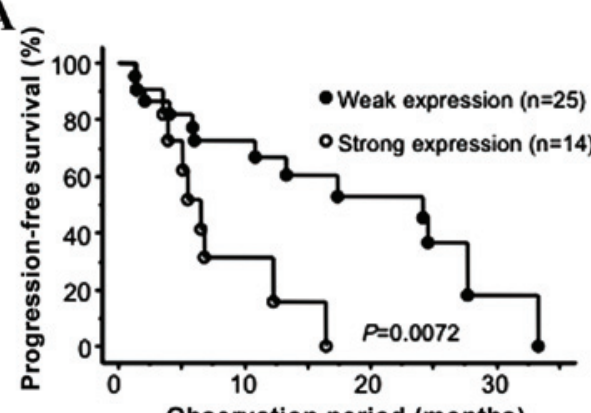

B

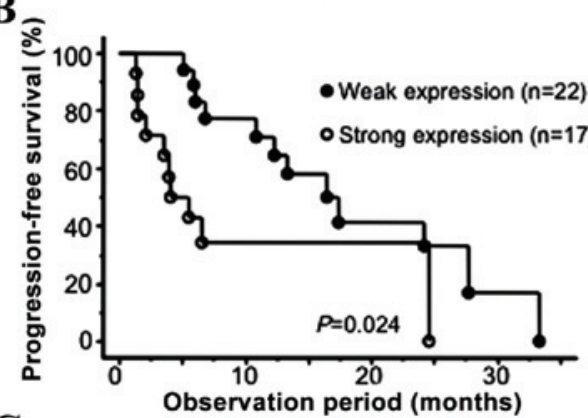

$\mathrm{C}_{\text {○ }}$

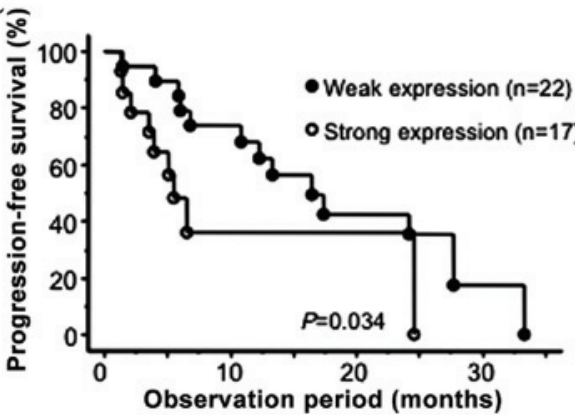

D

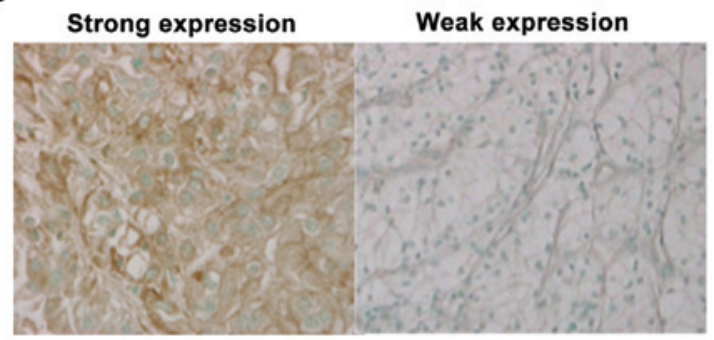

$\mathbf{E}$

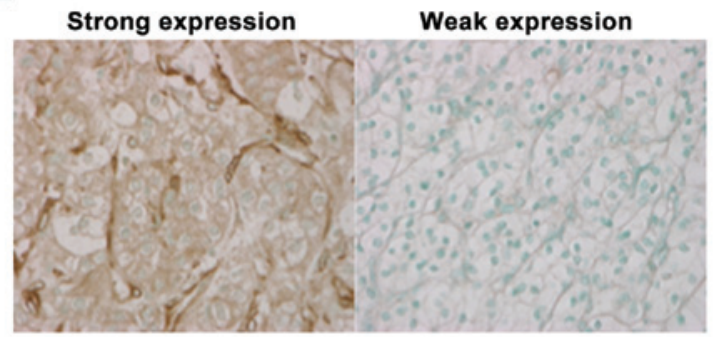

$\mathbf{F}$

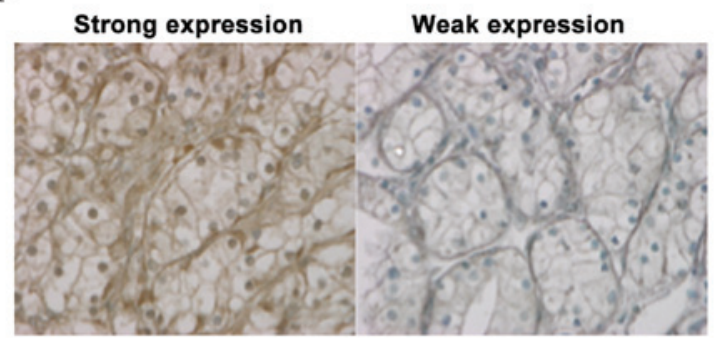

Figure 2. Comparison of the progression-free survival of the patients with metastatic clear cell renal cell carcinoma treated with sunitinib regarding the expression levels of (A) GLI2, (B) VEGFR-1 and (C) VEGFR2. Representative immunohistochemical images (magnification, x200) of radical nephrectomy specimens stained for (D) GLI2, (E) VEGFR-1 and (F) VEGFR-2. VEGF, vascular endothelial growth factor receptor.

present study was the first to report the prognostic value of a Hedgehog signaling-related protein (GLI2) in m-ccRCC patients receiving sunitinib.

In addition to the 3 molecular markers, the MSKCC and baseline CRP levels were also significantly correlated with PFS on univariate analysis. Multivariate analysis of these 5 parameters compared with the outcome of the $39 \mathrm{~m}$-ccRCC patients revealed a significant correlation between the expression levels of GLI2 and PFS, indicating the independent prognostic value of GLI2. GLI2 was initially regarded as having essential functions as an effector of the Hedgehog signaling pathway, while a number of studies have demonstrated the ubiquitous induction of GLI2 by TGF- $\beta$, resulting in the enhanced development of solid tumors (27-29). For instance, the overexpression of GLI2 in mouse skin using keratin 5 promoter was shown to be sufficient to initiate basal cell carcinomas (28), whereas GLI2 knockdown in prostate cancer cells delayed the growth of xenograft tumors and enhanced their sensitivity to chemotherapeutic agents (29). However, the current knowledge on the effect of GLI2 expression on the phenotype of RCC remains limited and the results of the present study should be confirmed in another cohort with a prospective setting.

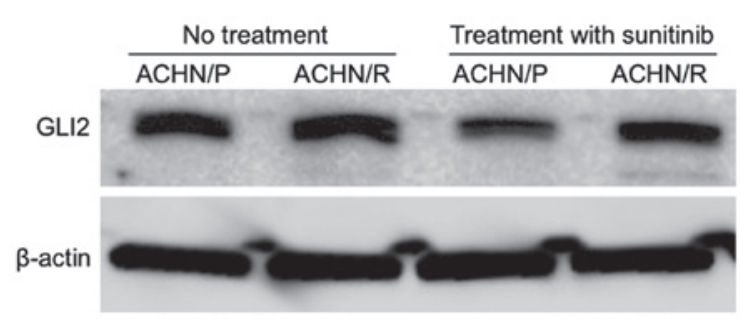

Figure 3. Western blot analysis evaluating the expression levels of GLI2 and $\beta$-actin in ACHN/P and ACHN/R treated with or without $5 \mu \mathrm{M}$ sunitinib. ACHN/P, parental ACHN glioma cell line; ACHN/R, ACHN cells with acquired sunitinib resistance.

It is of interest to investigate whether GLI2 expression in RCC cells mediates the acquisition of a resistant phenotype to sunitinib. Several previous studies have assessed the mechanisms underlying the acquired resistance of RCC cells to sunitinib $(17,30,31)$. In the present study GLI2 expression was maintained in sunitinib-resistant cells, but was decreased in parental cells following culture in the presence of sunitinib. This finding suggested the possible involvement of GLI2 expression in the resistance of RCC cells to sunitinib. Further experiments are required to determine the precise mechanism 
of the acquired resistance involving GLI2 regulation in RCC cells. Taken together, it may be worthwhile examining the role of additional treatment with an agent capable of inactivating GLI2, such as NVP-LDE225 (23), to overcome resistance to sunitinib in RCC patients.

Of note, the present study had certain limitations. First, it was a retrospective study and the cohort of 39 consecutive patients with m-ccRCC treated with sunitinib was not of sufficient size to draw definitive conclusions, particularly regarding their prognosis. Furthermore, the expression levels of the molecular markers was assessed in radical nephrectomy specimens only; however, it may have been suitable to also examine their expression in metastatic tissues to obtain results more closely reflecting the clinical outcomes. Finally, the present study focused on only 9 selected molecules as potential biomarkers for predicting the response of m-ccRCC to sunitinib; however, other molecules more significantly correlated with the prognosis of m-ccRCC patients receiving sunitinib may exist.

In conclusion, by simultaneous evaluation of several clinicopathological parameters along with expression levels of multiple Hedgehog signaling-related proteins as well as major molecular targets of sunitinib, only GLI2 expression was identified as an independent factor associated with PFS in m-ccRCC patients treated with sunitinib. Therefore, assessment of the expression levels of GLI2 in resected primary tumors in addition to conventional prognostic factors may aid in the careful selection of patients with m-ccRCC who are most likely to benefit from sunitinib treatment.

\section{Acknowledgements}

The present study was supported by JSPS KAKENHI (grant no. $15 \mathrm{~K} 20090)$.

\section{References}

1. Rini BI, Rathmell WK and Godley P: Renal cell carcinoma. Curr Opin Oncol 20: 300-306, 2008.

2. Parton M, Gore M and Eisen T: Role of cytokine therapy in 2006 and beyond for metastatic renal cell cancer. J Clin Oncol 24: 5584-5592, 2006.

3. Figlin R, Sternberg C and Wood CG: Novel agents and approaches for advanced renal cell carcinoma. J Urol 188: 707-715, 2012.

4. Gan HK, Seruga B and Knox JJ: Sunitinib in solid tumors. Expert Opin Investig Drugs 18: 821-834, 2009.

5. Mendel DB, Laird AD, Xin X, Louie SG, Christensen JG, Li G, Schreck RE, Abrams TJ, Ngai TJ, Lee LB, et al: In vivo antitumor activity of SU11248, a novel tyrosine kinase inhibitor targeting vascular endothelial growth factor and platelet-derived growth factor receptors: Determination of a pharmacokinetic/pharmacodynamic relationship. Clin Cancer Res 9: 327-337, 2003.

6. Motzer RJ, Hutson TE, Tomczak P, Michaelson MD, Bukowski RM, Rixe O, Oudard S, Negrier S, Szczylik C, Kim ST, et al: Sunitinib versus interferon alfa in metastatic renal-cell carcinoma. N Engl J Med 356: 115-124, 2007.

7. Hutson TE, Figlin RA, Kuhn JG and Motzer RJ: Targeted therapies for metastatic renal cell carcinoma: An overview of toxicity and dosing strategies. Oncologist 13: 1084-1096, 2008.

8. Motzer RJ, Bacik J, Murphy BA, Russo P and Mazumdar M: Interferon-alfa as a comparative treatment for clinical trials of new therapies against advanced renal cell carcinoma. J Clin Oncol 20: 289-296, 2002.

9. Heng DY, Xie W, Regan MM, Warren MA, Golshayan AR, Sahi C, Eigl BJ, Ruether JD, Cheng T, North S, et al: Prognostic factors for overall survival in patients with metastatic renal cell carcinoma treated with vascular endothelial growth factor-targeted agents: Results from a large, multicenter study. J Clin Oncol 27: 5794-5792, 2009.
10. Su D, Singer EA and Srinivasan R: Molecular pathways in renal cell carcinoma: Recent advances in genetics and molecular biology. Curr Opin Oncol 27: 217-223, 2015.

11. Ruch JM and Kim EJ: Hedgehog signaling pathway and cancer therapeutics: Progress to date. Drugs 73: 613-623, 2013.

12. Karnofsky DA, Abelmann WH, Craver LF and Burchenal JH: The use of nitrogen mustards in the palliative treatment of carcinoma. With particular reference to bronchogenic carcinoma. Cancer 1: 634-656, 1948.

13. Greene FL, Page DL, Fleming ID, Fritz A, Balch CM, Haller DG and Morrow M (eds.): AJCC Cancer Staging Manual. 6th edition. Springer, New York, NY, 2002.

14. Kususda Y, Miyake H, Gleave ME and Fujisawa M: Clusterin inhibition using OGX-011 synergistically enhances antitumour activity of sorafenib in a human renal cell carcinoma model. Br J Cancer 106: 1945-1952, 2012.

15. Miyake H, Muramaki M, Kurahashi T, Takenaka A and Fujisawa M: Expression of potential molecular markers in prostate cancer: Correlation with clinicopathological outcomes in patients undergoing radical prostatectomy. Urol Oncol 28: 145-151, 2010.

16. Li J, Wu T, Lu J, Cao Y, Song N, Yang T, Dong R, Yang Y, Zang L, Du X and Wang S: Immunohistochemical evidence of the prognostic value of hedgehog pathway components in primary gallbladder carcinoma. Surg Today 42: 770-775, 2012.

17. Sakai I, Miyake H and Fujisawa M: Acquired resistance to sunitinib in human renal cell carcinoma cells is mediated by constitutive activation of signal transduction pathways associated with tumour cell proliferation. BJU Int 112: E211-E220, 2013

18. Gore ME, Szczylik C, Porta C, Bracarda S, Bjarnason GA, Oudard S, Hariharan S, Lee SH, Haanen J, Castellano D, et al: Safety and efficacy of sunitinib for metastatic renal-cell carcinoma: An expanded-access trial. Lancet Oncol 10: 757-763, 2009.

19. Miyake H, Miyazaki A, Harada K and Fujisawa M: Assessment of efficacy, safety and quality of life of 110 patients treated with sunitinib as first-line therapy for metastatic renal cell carcinoma: Experience in real-world clinical practice in Japan. Med Oncol 31: 978, 2014

20. Yuasa T, Takahashi S, Hatake K, Yonese J and Fukui I: Biomarkers to predict response to sunitinib therapy and prognosis in metastatic renal cell cancer. Cancer Sci 102: 1949-1957, 2011.

21. Miyake H, Nishikawa M, Tei H, Furukawa J, Harada K and Fujisawa M: Significance of circulating matrix metalloproteinase-9 to tissue inhibitor of metalloproteinases-2 ratio as a predictor of disease progression in patients with metastatic renal cell carcinoma receiving sunitinib. Urol Oncol 32: 584-588, 2014.

22. Dormoy V, Danilin S, Lindner V, Thomas L, Rothhut S, Coquard C, Helwig JJ, Jacqmin D, Lang H and Massfelder T: The sonic hedgehog signaling pathway is reactivated in human renal cell carcinoma and plays orchestral role in tumor growth. Mol Cancer 8: 123, 2009.

23. D'Amato C, Rosa R, Marciano R, D'Amato V, Formisano L, Nappi L, Raimondo L, Di Mauro C, Servetto A, Fulciniti $\mathrm{F}$, et al: Inhibition of Hedgehog signalling by NVP-LDE225 (Erismodegib) interferes with growth and invasion of human renal cell carcinoma cells. Br J Cancer 111: 1168-1179, 2014

24. Zhou J, Wu K, Gao D, Zhu G, Wu D, Wang X, Chen Y, Du Y, Song W, Ma Z, et al: Reciprocal regulation of hypoxia-inducible factor $2 \alpha$ and GLI1 expression associated with the radioresistance of renal cell carcinoma. Int J Radiat Oncol Biol Phys 90: 942-951, 2014.

25. Deprimo SE, Bello CL, Smeraglia J, Baum CM, Spinella D, Rini BI, Michaelson MD and Motzer RJ, et al: Circulating protein biomarkers of pharmacodynamic activity of sunitinib in patients with metastatic renal cell carcinoma: Modulation of VEGF and VEGF-related proteins. J Transl Med 5: 32, 2007.

26. Kontovinis LF, Papazisis KT, Touplikioti P, Andreadis C, Mouratidou D and Kortsaris AH: Sunitinib treatment for patients with clear-cell metastatic renal cell carcinoma: Clinical outcomes and plasma angiogenesis markers. BMC Cancer 9: 82, 2009

27. Javelaud D, Alexaki VI, Dennler S, Mohammad KS, Guise TA and Mauviel A: TGF- $\beta /$ SMAD/GLI 2 signaling axis in cancer progression and metastasis. Cancer Res 71: 5606-5610, 2011.

28. Grachtchouk M, Mo R, Yu S, Zhang X, Sasaki H, Hui CC and Dlugosz AA: Basal cell carcinomas in mice overexpressing Gli2 in skin. Nat Genet 24: 216-217, 2000. 
29. Narita S, So A, Ettinger S, Hayashi N, Muramaki M, Fazli L, Kim Y and Gleave ME: GLI2 knockdown using an antisense oligonucleotide induces apoptosis and chemosensitizes cells to paclitaxel in androgen-independent prostate cancer. Clin Cancer Res 14: 5769-5777, 2008.

30. Huang D, Ding Y, Zhou M, Rini BI, Petillo D, Qian CN, Kahnoski R, Futreal PA, Furge KA and Teh BT: Interleukin-8 mediates resistance to antiangiogenic agent sunitinib in renal cell carcinoma. Cancer Res 70: 1063-1071, 2010.
31. Hammers HJ, Verheul HM, Salumbides B, Sharma R, Rudek M, Jaspers J, Shah P, Ellis L, Shen L, Paesante S, et al: Reversible epithelial to mesenchymal transition and acquired resistance to sunitinib in patients with renal cell carcinoma: Evidence from a xenograft study. Mol Cancer Ther 9: 1525-1535, 2010. 